\title{
Esofagectomía transhiatal laparoscópica
}

\author{
Laparoscopic transhiatal esophagectomy \\ Juan Antonio López Corvalá, ${ }^{*}$ Emir Alamea Corral, ${ }^{\ddagger}$ Narda Carrera Laureán, ${ }^{\ddagger}$ \\ Jesús Arturo Medina Ramírez, ${ }^{\ddagger}$ Juan Antonio López Prida ${ }^{\ddagger}$ \\ * Jefe de Departamento de Cirugía de Mínimo Acceso y Cirugía Bariátrica. \\ ₹ Cirujano General en adiestramiento en Cirugía de Mínimo Acceso y Cirugía Bariátrica. \\ Hospital Ángeles Tijuana, Baja California.
}

RESUMEN

Introducción: La acalasia es una enfermedad con una incidencia de uno en cada 100,000 habitantes. Su tratamiento más efectivo es la cardiomiotomía de Heller $(\mathrm{CH})$, siendo su complicación más frecuente el reflujo gastroesofágico (RGE), el cual puede llevar a estenosis esofágica. Caso clínico: Se presenta paciente de sexo masculino de 26 años de edad con antecedente de $\mathrm{CH}$ y funduplicatura tipo Dor por acalasia. Después de 10 años presenta estenosis esofágica de origen péptico, la cual no se resuelve mediante dilataciones esofágicas endoscópicas. Se realizó esofagectomía transhiatal con ascenso gástrico por abordaje de mínimo acceso. El objetivo fue efectuar la descripción del caso, su evolución y resultados así como realizar una revisión de los antecedentes y de la situación actual de la esofagectomía con abordaje de mínimo acceso para el manejo de la estenosis esofágica benigna; en este caso como complicación secundaria a RGE en el paciente con acalasia. Conclusión: El abordaje laparoscópico transhiatal es factible en los casos de estenosis esofágica benigna que requieren esofagectomía, es seguro y aporta todas las ventajas de la cirugía de mínimo acceso sin aumentar la morbilidad.

Palabras clave: Esofagectomía, transhiatal, mínimo acceso, acalasia, estenosis esofágica, miotomía.

\section{ABSTRACT}

Introduction: Achalasia is a disease that affects 1 in 10,000 inhabitants. The most effective treatment for this condition is Heller's Cardiomyotomy which may result in gastroesophageal reflux and in turn, esophageal stenosis. Case Study: We received a 26 year old male patient with a history of Laparoscopic Heller's Cardiomiotomy and Dor Fundoplication. 10 years after his treatment, he presented peptic esophageal stricture with poor response to endoscopic esophageal dilations. A transhiatal esophagectomy was performed with laparoscopic gastric ascent. The aim of this paper is to describe the case and the patient's evolution and results; along with a review of the history and current status of minimally invasive esophagectomy as a treatment for benign esophageal stenosis. Conclusion: A transhiatal laparascopic approach is feasible in benign esophageal stenosis cases that require esophagectomy. It is considered safe and offers the advantages that minimally invasive surgery has to offer without increasing its morbidity.

Keywords: Esophagectomy, transhiatal, minimally invasive, achalasia, esophageal stenosis, miotomy.

\section{INTRODUCCIÓN}

En la actualidad el abordaje laparoscópico se ha posicionado como el estándar de oro en la mayoría de los procedimientos quirúrgicos, sin ser la excepción la esofagectomía transhiatal, ya que una de sus principales ventajas es que permite mejor visualización de las estructuras mediastínicas y mejor movilización esofágica. ${ }^{1}$

Existen estudios comparativos entre el abordaje laparoscópico y abierto para este procedimiento cuyos resul-

Recibido: 23/09/2019. Aceptado: 13/01/2020.

Correspondencia: Dr. Juan Antonio López Corvalá. E-mail: drcorvala@grupolap.com

Citar como: López CJA, Alamea CE, Carrera LN, Medina RJA, López PJA. Esofagectomía transhiatal laparoscópica. Rev Mex Cir Endoscop. 2020; 21 (2): 100-103. https://dx.doi.org/10.35366/98914 
tados favorecen a la cirugía laparoscópica como menor pérdida sanguínea, disminución en los días de estancia en la unidad de cuidados intensivos, menos complicaciones pulmonares y en caso de patología maligna mayor margen libre de tumor. ${ }^{2}$

La esofagectomía generalmente se realiza para resecar el cáncer de esófago; sin embargo, existen otras indicaciones para la misma, incluido el esófago de Barrett con displasia de alto grado y algunas enfermedades benignas como acalasia en estado terminal, ${ }^{3}$ perforación del esófago, neoplasia benigna, lesión cáustica severa y estenosis péptica. ${ }^{4,5}$ Es un procedimiento quirúrgico complejo que desafía al cirujano, ya que requiere de una amplia disección cervical, torácica y abdominal, restituyendo la continuidad del tubo digestivo mediante ascenso gástrico o transposición colónica. Cabe mencionar que el abordaje laparoscópico transhiatal evita la toracotomía, siendo una de las grandes ventajas. ${ }^{6}$

La primera resección exitosa del esófago torácico se reportó en 1913 por Torek, desde entonces se han descrito diferentes técnicas con múltiples accesos para resecar el esófago. En la década de los 90 con la aparición de la cirugía endoscópica se inician las primeras esofagectomías laparoscópicas asistidas mediante toracoscopía. En 1995, DePaula y colaboradores describen la primera esofagectomía totalmente laparoscópica por vía transhiatal. ${ }^{4} \mathrm{~A}$ partir de esta fecha se empieza a replicar el abordaje con buenos resultados a nivel mundial. ${ }^{7,8}$

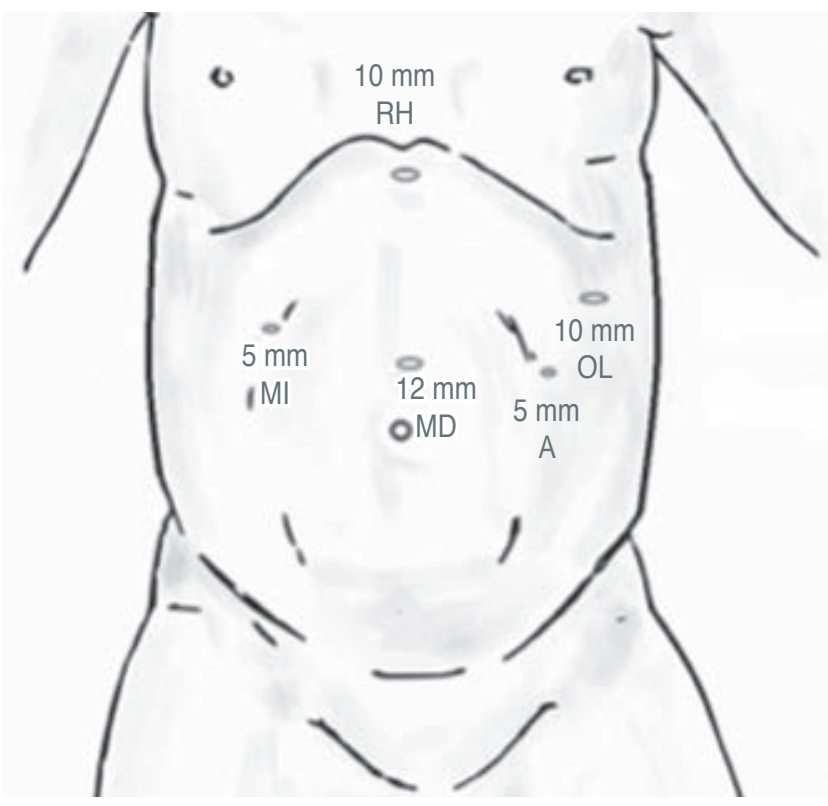

Figura 1: Colocación de puertos.

$\mathrm{MI}=$ mano izquierda del cirujano, $\mathrm{MD}=$ mano derecha del cirujano, $\mathrm{A}=$ ayudante, $\mathrm{OL}$ = óptica de laparoscopía, $\mathrm{RH}=$ retractor hepático

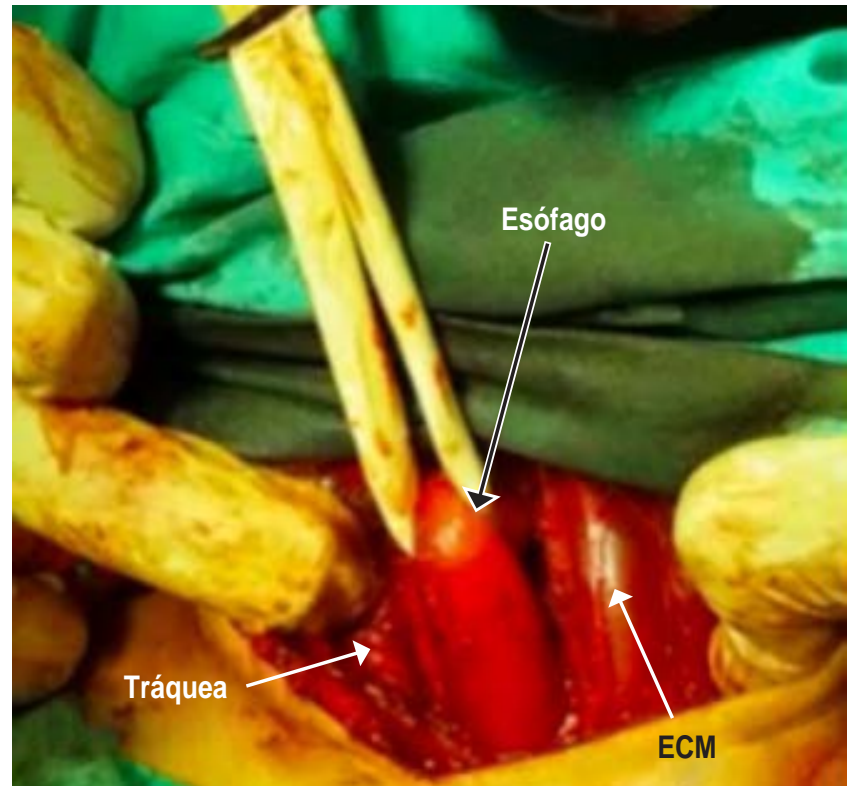

Figura 2: Disección de esófago cervical.

$\mathrm{ECM}=$ esternocleidomastoideo.

El objetivo de este estudio es presentar un caso poco frecuente: Acalasia complicada con estenosis severa de la unión esófago-gástrica (UEG) secundaria a reflujo gastroesofágico (RGE) y su tratamiento mediante esofagectomía por abordaje laparoscópico transhiatal.

\section{REPORTE DE CASO}

Paciente de sexo masculino de 26 años de edad con diagnóstico de acalasia con severa disfagia a líquidos en 2008. En 2009 se intervino quirúrgicamente de cardiomotomía de Heller $(\mathrm{CH})$ con funduplicatura tipo Dor con buena respuesta.

A partir de 2016 presenta disfagia a alimentos sólidos. En 2018 se suma disfagia a líquidos y pérdida de peso, con estudio de esofagograma y endoscopía que muestra disminución de la luz esofágica en su tercio inferior, por lo que fue manejado con abordaje endoscópico y dilatación esofágica en dos ocasiones, presentando mejoría relativa permitiendo el paso de líquidos. Meses después, por recurrencia de la sintomatología se realiza una tercera endoscopía, en la cual se identificó estenosis péptica por estudio histopatológico en tercio distal del esófago con disminución de la luz esofágica de $2 \mathrm{~mm}$ que no permite el paso de la sonda de dilatación. Por estudio radiológico contrastado se documentó sitio y longitud de la estenosis de $5 \mathrm{~cm}$.

El paciente es referido a nuestra consulta para manejo quirúrgico. Se mejora su estado nutricional, programándose para esofagectomía con ascenso gástrico y anastomosis 
esófago-gástrica con abordaje transhiatal laparoscópico (Figura 1) y cervical (Figura 2).

\section{DISCUSIÓN}

El abordaje laparoscópico para la esofagectomía requiere de una curva de aprendizaje amplia; sin embargo, a pesar de su dificultad técnica, no se ha reportado mayor incidencia de complicaciones en comparación con el abordaje abierto. ${ }^{9}$ Se ha demostrado una recuperación rápida con reducción de los días de estancia hospitalaria y reintegración más rápida a las actividades de la vida diaria. ${ }^{10}$ Debe realizarse en centros con experiencia que cuenten con el personal especializado en el manejo global que implica este procedimiento (anestesiólogos, terapia intensiva y cirujanos especializados con el procedimiento). ${ }^{11}$ Dentro de los riesgos de este tipo de cirugía existen estudios comparativos entre abordaje laparoscópico y abierto cuyos resultados favorecen al abordaje laparoscópico como menor pérdida sanguínea, disminución en los días de estancia en la unidad de cuidados intensivos, menos complicaciones pulmonares y en caso de patología maligna mayor índice de márgenes libres de tumor (Figura 3). ${ }^{10}$

Como parte de las posibles complicaciones se encuentran malnutrición y fuga de la anastomosis esofagogástrica, por lo cual algunos autores recomiendan la colocación de una sonda de yeyunostomía que proporcionaría una vía de alimentación. Existen complicaciones menores y mayores relacionadas a este procedimiento (infección del sitio de inserción, oclusión intestinal) con tasas de reoperación de hasta $2 \%$, por lo cual no se recomienda su uso rutinario. ${ }^{12}$

\section{Figura 3:}

Esófago resecado.

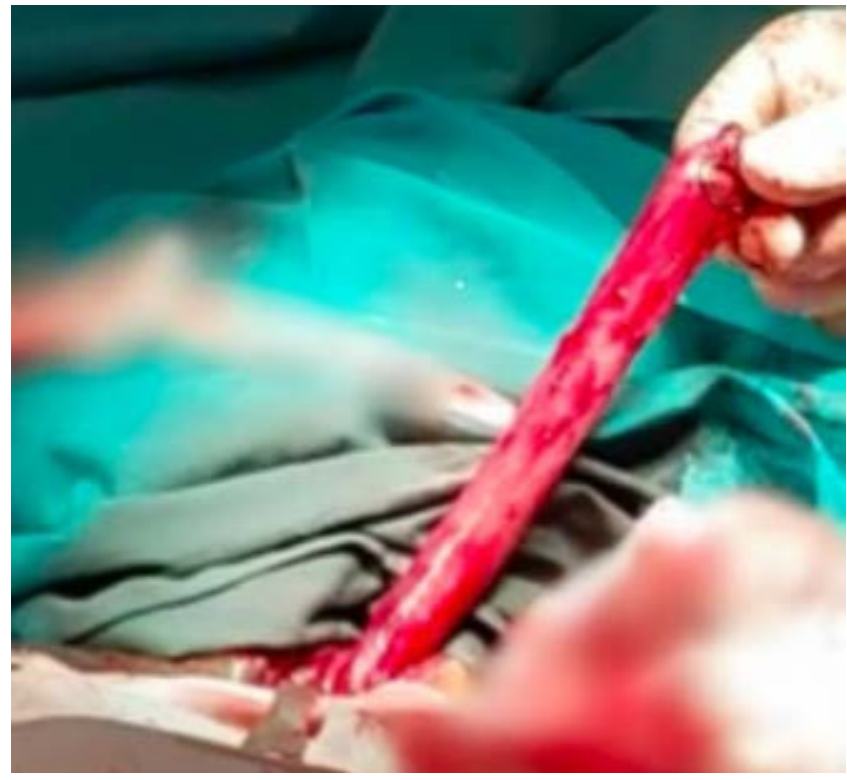

Figura 4: Extracción del esófago.

En este caso en particular se decidió colocar sonda de yeyunostomía para asegurar un aporte nutricional óptimo. Además, en caso de presentar fuga anastomótica, se cuenta con una vía de alimentación alterna.

La principal ventaja de la esofagectomía laparoscópica transhiatal es la mejor visualización de las estructuras mediastínicas, esta operación minimiza la posible inestabilidad hemodinámica frecuentemente encontrada por el uso del retractor y disección manual en la movilización esofágica (Figura 4). ${ }^{6}$

La cirugía robótica con la cual contamos en nuestro hospital facilita la cirugía de mínimo acceso al mejorar la vista del campo quirúrgico y aumentar el rango de movimiento de los instrumentos, incrementando las capacidades del cirujano. Reportes muestran que la cirugía laparoscópica asistida por robot es superior a la esofagectomía abierta en términos de complicaciones posoperatorias, duración de la estancia hospitalaria y calidad de vida. ${ }^{13}$ Acorde a los resultados obtenidos a nivel mundial la cirugía robótica debe ser contemplada para futuros casos.

\section{IIC.OYO / CONCLUSIÓN}

El tratamiento de elección de la acalasia en fase terminal y/o con estenosis esofágica por RGE no tratables mediante endoscopía es la esofagectomía en pacientes aptos para cirugía mayor.

El abordaje transhiatal laparoscópico es el procedimiento de elección por su baja morbimortalidad y buenos resultados. Además ofrece los beneficios de la cirugía de mínimo 
acceso (menor dolor posoperatorio, estancia hospitalaria más corta y una pronta reintegración a las actividades de la vida diaria), una disminución en complicaciones pulmonares, sangrado y días de estancia en $\mathrm{UCl}$. Consideramos que en los casos que sea posible, debe optarse por este abordaje quirúrgico, siempre y cuando se cuente con el equipo y personal calificado.

Es importante mencionar que la cirugía robótica representa una nueva alternativa para este tipo de casos, ya que incrementa las capacidades del cirujano, la visión del campo quirúrgico y la movilidad de los instrumentos, lo que influye en mejores resultados para los pacientes.

\section{REFERENCIAS}

1. Spechler SJ. Achalasia: pathogenesis, clinical manifestations, and diagnosis. UpToDate. 2018.

2. Salvador R, Pesenti E, Gobbi L, Capovilla G, Spadotto L, Voltarel G et al. Postoperative gastroesophageal reflux after laparoscopic Heller-Dor for achalasia: true incidence with an objective evaluation. J Gastrointest Surg. 2017; 21: 17-22.

3. Aiolfi A, Asti E, Bonitta G, Bonavina L. Esophagectomy for end-stage achalasia: systematic review and meta-analysis. World J Surg. 2018; 42: 1469-1476.

4. Mormando J, Barbetta A, Molena D. Esophagectomy for benign disease. J Thorac Dis. 2018; 10: 2026-2033.

5. Treitl D, Grossman R, Ben-David K. Esophagectomy for failed anti-reflux therapy: indications, techniques, and outcomes. In: Fisichella PM. Failed anti-reflux therapy. Springer, Cham. 2017, pp. 131-137.
6. Valentí V, Fares R, Reynolds N, Cohen P, Theodoro N, Martínez-Isla A. Esofagectomía transhiatal por vía abierta y vía laparoscópica para el cáncer de esófago: análisis de los márgenes de resección y ganglios linfáticos. Cirugía Española. 2008; 83: 24-27.

7. Murthy RA, Clarke NS, Kernstine Sr. KH. Minimally invasive and robotic esophagectomy: a review. Innovations (Phila). 2018; 13: 391-403.

8. Moonen A, Annese V, Belmans A, Bredenoord AJ, Des Varannes SB, Costantini $M$ et al. Long-term results of the European achalasia trial: a multicentre randomised controlled trial comparing pneumatic dilation versus laparoscopic Heller myotomy. Gut. 2016; 65: 732-739.

9. Fontan AJA, Batista-Neto J, Pontes ACP, Nepomuceno MC, Muritiba TG, Furtado RS. Minimally invasive laparoscopic esophagectomy vs transhiatal open esophagectomy in achalasia: a randomized study. ABCD Arq Bras Cir Dig. 2018; 31: e1382.

10. Maas KW, Biere SS, Scheepers JJ, Gisbertz SS, Van der Peet DL, Cuesta MA. Laparoscopic versus open transhiatal esophagectomy for distal and junction cancer. Rev Esp Enferm Dig. 2012; 104: 197-202.

11. Medina H, Cortés R, Ramos GG, Quezada C, Flores C, Orozco H. Esofagectomía transhiatal. Experiencia en 20 años en el Instituto Nacional de Ciencias Médicas y Nutrición "Salvador Zubirán". Cir Gen. 2009; 31: 87-90.

12. Weijs TJ, Van Eden HW, Ruurda JP, Luyer MD, Steenhagen E, Nieuwenhuijzen GA, Van Hillegersberg R. Routine jejunostomy tube feeding following esophagectomy. J Thorac Dis. 2017; 9: S851-S860.

13. Meredith K, Blinn P, Maramara T, Takahashi C, Huston J, Shridhar R. Comparative outcomes of minimally invasive and robotic-assisted esophagectomy. Surg Endosc. 2009; 34: 814-820. 